21 Spitzer RL, Fleiss JL. A re-analysis of the reliability of psychiatric diagnosis. Br J Psychiatry 1974; 125: 341-7.

22 Kendell R, Jablensky A. Distinguishing between the validity and utility of psychiatric diagnoses. Am J Psychiatry 2003; 160: 4-12.

23 van Putten T, Crumpton E, Yale C. Drug refusal in schizophrenics and the wish to be crazy. Arch Gen Psychiatry 1976; 33: 1443-6.

24 Crisp AH, Gelder MG, Rix S, Meltzer HI, Rowlands OJ. Stigmatisation of people with mental illnesses. Br J Psychiatry 2000; 177: 4-7.

25 Corker E. Stigma caused by psychiatrists (letter). Br J Psychiatry 2001; 178: 379

26 Watson AC, Corrigan P, Larson JE, Sells M. Self-stigma in people with mental illness. Schizophr Bull 2007; 33: 1312-8.

27 Corrigan PW, Watson AC. Understanding the impact of stigma on people with mental illness. World Psychiatry 2002; 1: 16-20.

28 Sankar P, Jones NL. To tell or not to tell: primary care patients' disclosure deliberations. Arch Intern Med 2005; 165: 2378-83.
29 Sankar P, Mora S, Merz JF, Jones NL. Patient perspectives of medical confidentiality: a review of the literature. J Gen Intern Med 2003; 18: 659-69.

30 Angermeyer MC, Daumer R, Matschinger $\mathrm{H}$. Benefits and risks of psychotropic medication in the eyes of the general public: results of a survey in the Federal Republic of Germany. Pharmacopsychiatry 1993; 26: 114-20.

31 Double D. The limits of psychiatry. BMJ 2002; 324: 900-4.

32 Szasz TS. The myth of mental illness. Am Psychol 1960; 15: 113-8.

33 Sarkar SP. A British psychiatrist objects to the dangerous and severe personality disorder proposals. J Am Acad Psychiatry Law 2002; 30: 6-9.

34 American Psychiatric Association. The Principles of Medical Ethics with Annotations Especially Applicable to Psychiatry. APA, 2001.

35 Post JM. Ethical considerations in psychiatric profiling of political figures. Psychiatr Clin North Am 2002; 25; 635-46.

\title{
Medical psychotherapy: a specialty for now
}

\author{
Chris Mace, ${ }^{1}$ Kevin Healy ${ }^{2}$
}

The Psychiatrist (2011), 35, 301-304, doi: 10.1192/pb.bp.110.032599

\begin{abstract}
'University of Warwick; ${ }^{2}$ West London Mental Health NHS Trust

Correspondence to Kevin Healy (kevin.healy@wlmht.nhs.uk)

First submission 13 Sep 2010, final revision 15 Dec 2010, accepted 28 Jan 2011

Summary If many patients having multiple difficulties are to receive the integral and efficient psychotherapies they require, they need attention from psychiatrists who have specialised training in psychotherapy. This paper sets out the roles to which existing holders of the Certificate of Completion of Training have already moved to, as they work with patients, families, teams and organisations. The General Medical Council has recognised that the understanding of medical psychotherapists is also vital to the future clinical teaching of all psychiatric trainees. This paper summarises key components of the knowledge and roles of future specialists in medical psychotherapy. It recommends that the term 'medical psychotherapy' be used widely to clearly differentiate psychiatrists with this training and these responsibilities from non-medical psychotherapists.
\end{abstract}

Declaration of interest None.
Six psychiatric specialties are listed in the legislation that gave overall responsibility for psychiatric training to the Postgraduate Medical Education and Training Board. ${ }^{1}$ They will be familiar as the options for specialty training from year 4 (ST4): general psychiatry, child and adolescent psychiatry, forensic psychiatry, old age psychiatry, psychiatry of learning disability, and psychotherapy. Doubts were raised about the adequacy of this list as a map of 21st-century psychiatry when the issue of the number of Certificates of Completion of Training (CCTs) in psychiatry was considered during an abortive attempt at reform. ${ }^{2}$ Although the number of CCTs is now unlikely to change, the current wider range of faculties and sections within the Royal College of Psychiatrists provides a more accurate map of specialism within psychiatry.

\section{From psychotherapy to medical psychotherapy specialty}

Among the CCT-bearing specialties, one is unique. 'Psychotherapy' is not descriptive of the types of patients seen, as is the case with the other five psychiatric specialties, but is instead descriptive of the therapeutic activity undertaken. Psychotherapy is not limited to clinical work that is only undertaken by psychiatrists. Indeed, as the importance of psychological therapies in the care of people with mental health problems grows, this is increasingly the case. As the range of professionals providing psychotherapy expands, so does the scope for confusion concerning the different expertise of the professionals delivering it. After due internal discussion and endorsement, the College has applied to the Department of Health for the psychiatric 
specialty of psychotherapy to be renamed 'medical psychotherapy'. Likewise, the College's faculty will be known as the Faculty of Medical Psychotherapy. Changing the name will more clearly differentiate this area of psychotherapeutic practice from all others. No substantive changes in the role of consultant psychiatrists in psychotherapy are being proposed with this name change. The new name reflects changes in the scope of the specialty that have already taken place and deserve wider recognition.

\section{Change of name - rationale and significance}

What then are these changes? The first to note is that the working role of most medical psychotherapists has become more like that of other consultant psychiatrists than many people imagine. The role is essentially a clinical one. In carrying it out the medical psychotherapist works not only as a highly trained therapist, but typically leads a multidisciplinary team providing care for people who are sick and/or chronically disabled. Like other effective psychiatrists, he or she not only uses advanced skills in diagnosis, formulation and risk assessment, but is familiar with physical and social treatments in order to design and monitor complex interventions that include evidence-based psychotherapies. To be effective, these packages of care need to be sensitive to the physical and psychological needs of an individual, to their illness history, and to the clinical and social setting in which psychotherapies are delivered. This role inevitably includes working with patients in crisis and emergency situations. To do all this demands proficiencies core to the training of future medical psychotherapists that are not integral to the training of non-medical psychotherapists. As in any other psychiatric specialty, legal responsibilities for assessment and treatment may be assumed. In these respects and more, the name medical psychotherapy encapsulates current training and working realities far better than psychotherapy alone.

A second change to note is a distinctive sense of cohesion now growing among medical psychotherapists. Only a few years ago the professional identity of medical psychotherapists was grounded in affinities with nonmedical colleagues through their theoretical and practical models of working. Those trained as cognitive-behavioural, psychoanalytic/psychodynamic, integrative or systemic therapists shared significant theoretical underpinnings and clinical practices. Although these distinctions continue to matter a good deal, they are seen to recede alongside a sense that medical psychotherapists of all kinds are pragmatically concerned with meeting the clinical needs of people whose presentations are psychologically and medically complex. Tailoring therapeutic strategies to the needs of these patients involves intricate analysis of relevant risks and willingness to apply an extensive knowledge of psychotherapeutic practices responsively. This clinical common ground sits alongside other areas of responsibility shared by specialists in medical psychotherapy which are becoming more explicit. ${ }^{3}$ These include the coordination of training of all psychiatrists in psychotherapeutic formulation and skills; being able to work across teams so that other mental health professionals feel more capable of offering psychological interventions within their traditional roles; and leading a group of trained therapists who may be relatively unfamiliar with culture within the National Health Service (NHS), with evidence-based practice and with therapeutic modalities beyond the first modality they have trained in.

Assuming the change of name is sensible, why is this the moment to make it? The answer comes down to three 'R's. In no particular order, these are: regulation, resurgence and recognition.

\section{Regulation}

While medical psychotherapy has been evolving, the professional base of the remainder of UK psychotherapy has been quickly developing too. Training opportunities have been created that are linked to guaranteed jobs and open to people from all professional backgrounds and none. The Improving Access to Psychological Therapies (IAPT) programme across primary care is probably the clearest example of this. ${ }^{4}$ The growth of the therapeutic workforce adds to the existing pressure to bring non-medical psychotherapists of all persuasions under a formal regulatory framework. Despite the original medical roots of psychotherapy, many people now refer to psychotherapy as a 'new' profession. Moves to regulate non-medical psychotherapists (and counsellors) through an existing statutory regulator, the Health Professions Council (HPC), are the culmination of this process. These professions will be recognised separately from another group, mainly clinical and counselling psychologists, who often provide psychological treatments and who are already regulated by the HPC as 'practitioner psychologists'.

This process is not yet complete. Earlier discussions on the HPC's forum, to which medical practitioners have not been invited, involved regulation of both the 'diagnosis' of 'severe mental disorders' and treatment of their 'causes' being proposed as the characteristic proficiencies of psychotherapists (as opposed to counsellors). ${ }^{5}$ In subsequent consultation, psychotherapists, who generally pride themselves on working outside a 'medical model', were quick to affirm that diagnosis was the province of psychiatrists rather than psychotherapists. To date, a satisfactory specification of the core characteristics of the practice of a (non-medical) profession of psychotherapy remains elusive. Among the many views on the remit of the profession, the chair of the UK Council for Psychotherapy publicly asserted that psychotherapists 'are not a health profession'. ${ }^{6}$

However the debate on the nature and scope of psychotherapy as practised by non-medical therapists is resolved, it is clear that, very shortly, two regulators will be involved: the General Medical Council (GMC) for medical psychotherapists and the HPC for counsellors and all other psychotherapists. All regulation needs to ensure that the interests of the public are safeguarded effectively. In this situation, the public should not only have the reassurance that the professional they are seeing is complying with clear standards, but know which regulator the professional is answerable to. As the GMC has been regulating psychiatrists specialising in psychotherapy for some time, any confusion that results from other psychotherapists being answerable 
to the HPC will be minimised by having medical psychotherapy as the activity for which the GMC remains responsible.

\section{Resurgence}

The sense of coming together in the service of individuals with similar problems, irrespective of differences in psychotherapeutic models and philosophies, helps members of our specialty work together. Medical psychotherapists are currently working around several common clinical and educational issues. These include: the dangers and risks of inexpert interventions; using understanding of brain, body and autonomic nervous system functioning in accounting for the clinical consequences of multiple traumas (details available from K.H. on request); the place of family in personal psychotherapy; the economic implications of intensive and team-based treatments; the influence of training on boundary violations; the need for methodical investigation alongside the use of evidence-based practice; ${ }^{7}$ the development of expertise in psychological and psychosomatic dangers of personality disorder; ${ }^{8}$ and idiosyncratic responses to therapy in the presence of commonly prescribed psychotropic medications. As this programme takes further shape, medical psychotherapy as a discipline will have an increasingly expanding, distinctive and fresh knowledge base. A first textbook that sets this out has already been commissioned by Oxford University Press and is due for publication in 2011. ${ }^{9}$

Alongside a change in how medical therapists work and think, there needs to be resurgence in their numbers too. It is important for the specialty of medical psychotherapy to be present and actively contributing in each and every mental health service. This contribution will be felt in the efficiency and effectiveness of a service and in the confidence and safety experienced by staff in working with sets of patients who are especially demanding or ambiguous in their presentation. This required expansion cannot rely upon the emergence of fully-fledged specialists from NHS psychotherapy training schemes alone. It will also depend upon the recognition of the expertise of consultants in other psychiatric specialties who already have significant training in psychotherapy and other relevant experiences as medical psychotherapists. Changes in regulation will make a contribution here. As GMC revalidation moves towards a process of accrediting specialists in relation to the work that they are actually doing, more trained psychiatrists are likely to be brought within the medical psychotherapy umbrella on account of their actual work, and to have this recognised in future revalidation.

\section{Recognition}

The role and contributions of medical psychotherapy are already recognised within the Royal College of Psychiatrists, but three other kinds of recognition are important. The first is recognition from non-medical colleagues practising psychotherapy under the regulations of the HPC. Medical psychotherapists are likely to remain heavily involved in the supervision and training of other therapists and in turn will likely need to call on the involvement of non-medical psychotherapists in the clinical training of psychiatrists. Medical psychotherapists, however, need to be vocal and seek active engagement with government and with policy-making bodies. They need to ensure adequate representation on all relevant statutory and regulatory bodies as these evolve.

The second kind of recognition applies to employers within the NHS and in the private and independent sector. All such employers will want value for patients and for services provided from any investment in individual professionals. They need to be in no doubt that medical psychotherapists are first and foremost medical professionals and as such can provide value for the investment in them as a resource to patients, services, colleagues, employers and commissioners. This investment needs to be protected and nurtured by conditions of employment that include study leave arrangements, which facilitate learning and development through contact with medical psychotherapists and other colleagues who may be experts by training or by their lived experience. Local professional management arrangements need to support the development of medical psychotherapists as individual professionals who can make valued contributions to clinical services, to education and training, to research and evaluation, to a real involvement of service users, their friends and families as partners in their own treatments and in service developments, and to the public health agenda locally.

We recognise that individual medical psychotherapists in their own localities, and the Royal College of Psychiatrists' Psychotherapy Faculty nationally, face a challenge in persuading employers of the value of employing consultant medical psychotherapists in the present economic climate. Perhaps then the third group to seek recognition from remains our own profession. Fully recognising and valuing where our greatest expertise lies may be essential in terms of making other things happen. We need to recognise what interventions, seen as necessary and valued by patients, commissioners and provider organisations, we are best trained and equipped by experience to deliver. In all areas of our practical work with patients, teams and organisations we need to be seen to keep developing our own skills, alongside the developments we facilitate in others.

\section{Changes that will go with a change in name}

The term 'medical psychotherapy' will apply to all references to the specialty. It will be used in naming the training stream from specialty training ST4 and the CCT; in equivalence proceedings; in entries in the GMC's Specialist Register; and in workforce planning including professional census taking. It will become the standard term for health systems within and beyond the NHS. Any psychiatrist already registered as a specialist in psychotherapy will automatically be recognised as registered as a specialist in medical psychotherapy. Use of the term will also help in the identification of the resources each mental health service has, in distinction from other kinds of psychological therapy provision. 
Other possible changes may not be intended, but may still arise as a result of the change in name. One is that a greater division might be felt between those consultants who immediately become medical psychotherapists by virtue of their listing on the Specialist Register, and other psychiatrists practising substantial amounts of psychotherapy, possibly privately, who also see themselves as medical psychotherapists despite not being listed as such. Their situation with respect to regulation is admittedly more complicated. Plans are being made to keep circumstances for dual regulation to a minimum.

Much of the discussion about renaming the specialty has understandably focused on the situation of specialists and colleagues who have a lot of training in psychotherapy theory and skills. However, the Psychotherapy Faculty retains a core role in the promotion of therapeutic thinking and action across all branches of psychiatry, before and after graduation. If there is any threat in the renaming of the specialty to keep focus on this important area of work, it will be short term only. We believe that psychotherapeutic practice in psychiatry will be more secure as representation of medical psychotherapy is established in each and every mental health service. In addition, its continuing intellectual growth along new avenues that are likely to interest psychiatrists less attracted by exclusive aspects of psychotherapy is most likely to win it greatest acceptance.

Finally, we welcome feedback from any colleagues interested in this work. We particularly welcome hearing from those of you who disagree with anything we have written. We wish to continue learning from and developing our work as individuals and as a faculty within the Royal College of Psychiatrists. Listening carefully with a learning mindset to the views of those who disagree with us is often one of the most powerful learning experiences in life.

\section{Acknowledgement}

Chris Mace sadly but courageously died after writing this article in November 2010. He is greatly missed by family, friends and colleagues.

\section{About the authors}

Chris Mace (deceased), formerly consultant psychotherapist, St Michael's Hospital, Warwick, and University of Warwick, past chair (2006-10), Psychotherapy Faculty, Royal College of Psychiatrists; Kevin Healy, lead clinician and consultant psychotherapist Cassel Hospital, Richmond, chair, Psychotherapy Faculty, Royal College of Psychiatrists, London, UK

\section{References}

1 Postgraduate Medical Education and Training Board. The General and Specialist Medical Practice (Education, Training and Qualifications) Order 2003. Statutory Instruments 2003; 1250.

2 Bhugra D, Jackson R. Education and training. In 2006 Annual Review: 6-7. Royal College of Psychiatrists, 2006.

3 Royal College of Psychiatrists. Role of the Consultant Psychiatrist in Psychotherapy (College Report CR139). Royal College of Psychiatrists, 2006

4 Mace C, Rowland N, Evans C, Schroder T, Halstead J. Psychotherapy professionals in the UK: expansion and experiment. Eur J Psychother Counsel 2009; 11: 131-40.

5 Health Professions Council. Draft Standards of Proficiency for Counsellors and Psychotherapists. HPC, 2009.

6 Samuels A. Therapy regulation. Times 2009; 12 December.

7 Gabbard GO. Psychodynamic Psychiatry in Clinical Practice (4th edn). American Psychiatric Publishing, 2005

8 Healy K. Living with personality disorder. New Associations 2010; 3.

9 Johnston J. Medical Psychotherapy: Specialist Handbook in Psychiatry. Oxford University Press, 2011 (in press). 